\title{
IDENTIFICATION OF EPIDURAL SPACE - WHICH TECHNIQUE SHOULD I ADOPT?
}

\author{
Akshaya.N.Shett \\ Fellow in Cardiac Anaesthesia, Dept of Cardiac Anaesthesia, KLESH Heart Foundation, \\ J.N.M.C. Belgaum- 590010, India.
}

*Corresponding author: aksnsdr@gmail.com

Key words: epidural anaesthesia, epidural space

\begin{abstract}
Regional anaesthesia, because of its safety and accuracy has an important role in modern anaesthetic practice. Neuraxial anaesthesia has a great impact on all surgical fields and expands the anaesthesiologist's armamentarium. Epidural anaesthesia becomes popular because of advantages like slower onset of action, controlled hypotension and bradycardia, segmental blockade, usefulness in neck and thoracic surgeries and also for the management of postoperative analgesia.

The ability of epidural anaesthesia to produce neuraxial anaesthesia and analgesia without dural puncture provides an attractive alternative to spinal anaesthesia. Improvements in equipment, drugs and techniques, has made it popular and a versatile anaesthetic technique.

Acceptance of epidural blockade is limited by the fear of failure, neurological complications or slow onset. The success lies on correct identification of epidural space. Hence, choosing a correct method is important. This article mentions various techniques available to identify epidural space that can be adapted.
\end{abstract}

Ever since Karl Koller gave the first clinical demonstration of the local anaesthetic properties of cocaine in $1884,{ }^{1}$ local and regional anaesthesia has erratic clinical application. Following this great milestone, regional anaesthesia became a widely used technique by anaesthesiologists, realising its advantages over general anaesthesia.

In October 1885, the New York City physician James Leonard Corning published an article under the title "Spinal Anaesthesia and local medication of the spinal cord", an event regarded for many years as the first spinal blockade. As the lumbar puncture had not been reported before, even the appearance of cerebrospinal fluid was not mentioned; despite the large dosages of cocaine that were injected, the onset of action was slow and the extent of analgesia limited. Most likely, the injections were made into the peridural space and hence Corning was considered as the pioneer of peridural anaesthesia.

French physicians Jean Athanase Sicard and Fernand Cathelin independently did research on epidural analgesia. Cathelin was the first person to report on experiences by blocking the last sacral and coccygeal nerves by an anaesthetic solution in 1901. Later on it was applied to clinical surgery by Pages in 1921 and by Dogliotti and Aburel in 1931 and popularised by Massey Dawfin.

Since then different techniques have been explained by many researchers but still there exists no consensus as to which technique is superior, and individual providers currently use the technique with which they are most comfortable. The ideal technique for identifying the epidural space remains unclear. The author comprehensively discusses different techniques available for identification of epidural space and possible advantages and disadvantages of each technique, so that the reader can decide the technique to be adapted for his/her routine practice.

As regional anaesthesia has various advantages over general anaesthesia, it is preferred whenever applicable. Many of the 
surgeries are performed under central neuraxial blockade as a sole anaesthetic technique or as an adjunct to general anaesthesia. Amongst the central neural blockades epidural block is preferred over subdural block in day to day clinical anaesthetic practice because of added advantages.

Epidural anaesthesia has become an integral part of today's practice of anaesthesia. Present methods of identification of epidural space reflect a long process of change, largely in response to the limitations and complications of early techniques. Various techniques have been identified by many researchers since Pages described it in 1921 by the feel of giving way sensation as the needle passes through the spinal ligaments ${ }^{2}$. Ideal technique should be, easy to learn, highly sensitive and specific, cost effective, should not cause dural puncture or injure the nerves, more objective than subjective, should not affect the quality of anaesthesia, and have no effects on the haemodynamics.

\section{Loss of resistance technique:}

This was first explained in early $20^{\text {th }}$ century and is the most commonly used method till now by many anaesthesiologists. Sicard and Forestier used fluid as a medium for this technique in 1921. Dogliotti explained the principle behind it and popularized the technique $^{3}$. The advantages of this technique is that it is easy to learn, cost effective, and not cumbersome with high sensitivity and specificity. People who have experience in appreciating 'give way' feel can adopt this technique. It is not a very good technique for the beginners. Both air and fluid can be used as a medium for this technique but up-to-date the debate is going on as to which medium is superior. Air becomes the popular medium with some reasons. First is historical, as until the seventies, syringes were made from glass and were non-disposable. Fluid made the syringes sticky hence air replaced it by avoiding it. Disadvantages of using air are possibility of causing pneumocephalus 4,5 , headache ${ }^{5}$, cervical emphysema ${ }^{6}$, patchy block, and air embolism ${ }^{7}$. All these problems are encountered when the amount of air exceeds the standard limits. It is important to note that, air should not be used as a medium after an accidental dural puncture ${ }^{5}$. Air is a compressible medium and high pressure cannot be achieved when compared to noncompressible matter like fluid. With the availability of plastic syringes fluid gained popularity as a medium as it eliminated most of the problems of air as a medium. The main disadvantages with the use of saline are dilution of local anaesthetic agent affecting sensory blockade, and confusion with cerebrospinal fluid if accidental dural puncture occurs. Using more than $10 \mathrm{ml}$ of normal saline in epidural space does protect venous cannulation but at the expense of possible impairment of the quality of analgesia. Using $5 \mathrm{ml}$ of normal saline is associated with a lower incidence of intravascular catheter insertion and fewer unblocked segments ${ }^{8}$. Simple bedside testing for temperature, glucose, protein and $\mathrm{pH}$ accurately distinguished between CSF and saline used to identify the epidural space ${ }^{9}$. Saline as a medium has advantages if we select it especially in a parturient patient ${ }^{10}$. Some anaesthesiologists prefer using Lignocaine as a medium instead of normal saline as it was blamed for causing delayed onset of action of local anaesthetics ${ }^{11}$.

\section{Hanging drop technique:}

The principle in this technique is based on the fact that the pressure in the epidural space is sub atmospheric. Hence once loss of resistance is felt a drop of saline is put at the hub of the syringe. Advantage is that only a drop of saline is used and therefore has no effect on the quality of analgesia. In this technique the epidural needle is gripped by the thumb and index finger of both hands, with the ulnar border of the hands resting on the patient's back, allowing stability and control as the needle is advanced slowly through the ligamentum flavum ${ }^{12}$. Because of more negative pressure in thoracic region this is the preferred technique than for the lumbar region. Still this technique is rarely used. At the lumbar level the loss of resistance technique diminishes the risk of damaging dura mater compared with the hanging drop technique $^{13}$.

\section{Saline infusion technique}

As there is negative pressure in the epidural space the saline from the infusion set enters 
the epidural space. Baraka A reported this technique $^{14}$. The main advantages of this technique is that the needle can be gripped by both the hands and also directed according to the needs. It is easy to learn especially for beginners, has a high success rate, is cost effective, objective method as compared to loss of resistance technique which is subjective. Amount of saline entering will be minimal if micro infusion set is used hence does not affect the quality of analgesia. Saline which enters the space makes threading the catheter very easy, and small needles can be used. Disadvantages may include accidental dural puncture, and confusion with cerebrospinal fluid in such cases. Such disadvantages are minimal as both hands are used to guide the needle and controls the depth of entry hence chances of dural puncture will be minimal. There are chances of accidental entry of needle into epidural venous channels. This technique can be better utilized for thoracic epidurals, and even for the lumbar epidurals especially for the beginners.

\section{Dual technique:}

Dual technique for identification of epidural space uses loss of resistance technique with auditory amplification hence increasing the success rate ${ }^{18}$. It can be used in case of difficult anatomy, is cost effective, but a little cumbersome technique.

\section{Ultrasonography guided identification of epidural space:}

This is a newer technique, in which knowledge of ultrasound is used to identify the epidural space. Advantage of this method is accurate identification of epidural space including the depth of the epidural space, and is a better technique in case of obese patients. Disadvantages are that it is not cost effective; knowledge of Ultrasonography is mandatory, is a cumbersome technique and requires two operators. This method can be adopted by the obstetric anaesthesiologists, as ultrasonography machines are readily available and in case of difficult anatomy ${ }^{15}$. This method is mainly of academic interest with little practical application. Senior anaesthesiologists can utilize this technique to demonstrate epidural space anatomy to trainees.

\section{Compuflo Injection Pump for identification of epidural space:}

Here the epidural space was solely identified with the computerized injection pump. This technique is still under research. Advantage is that it is an objective technique, with identification of epidural space, lowering the incidence of side effects ${ }^{16}$. Disadvantages are that it is not a cost effective method.

Optical method for identification of epidural space:

A porcine model has been studied and introduced recently. Advantages are that it can be performed by a single operator, use a single wavelength; is a simple and sensitive technique and can be performed with inexpensive equipment and can be applied in the operating room or ward without radiation, enhance training efficacy because both the instructor and students can acquire the same information ${ }^{17}$. Disadvantages are that the model is still under trial, may not be cost effective in developing countries, trial on human subjects is required, as still there are chances of accidental dural puncture.

\section{Conclusion:}

Before making a premature conclusion that a particular technique is the cause of the failure to identify the epidural space or the cause of complications, it might also be important to consider the insertion skills of the provider and the ease or difficulty of the insertion procedure.

It is important to note that, anaesthesiologists should have the knowledge of most of the techniques and should use them regularly. There is a natural tendency for people to resist change. One who has performed loss of resistance with air successfully for a long time is not likely to switch to other techniques. Finally it is important to consider the easiness, safety, availability of resources, clinical situation before adopting any of the techniques.

\section{References:}

1. Odoms CB. A review of pages epidural anaesthesia with a report of 100 cases. New Orleans Medical and surgical Journal. 1936: 88: 618-27.

2. Pages F. Anestesia metamerica. Revista de sanidad Militar. 1921; 11; 351-65. 
3. Dogliotti AM. A new method of block anaesthesia. Segmental peridural spinal anaesthesia. American Journal of Surgery 1933: 20: 107-18.

4. Simopoulos T. and Peeters-Asdourian C. Pneumocephalus after Cervical Epidural Steroid Injection. Anaesthesia and Analgesia 2001: 92: 1576-1577.

5. Silvia Laviola, Maija Kirvela, Maria-Rosaria Spoto et al. Pneumocephalus with Intense Headache and Unilateral Pupillary Dilatation After Accidental Dural Puncture During Epidural Anesthesia for Cesarean Section Anesthesia Analgesia 1999:88: 582-3.

6. Carter MI. Cervical surgical emphysema following extradural analgesia. Anaesthesia 1984:39: 1115-6.

7. Naultly J. S. et al. Incidence of venous air embolism during venous catheter insertion. Anaethesiology 1982: 57: 410-412.

8. Shmuel Evron, Vladimir Gladkov. Daniel I. Sessler, et al Predistention of the Epidural Space Before Catheter Insertion Reduces the Incidence of Intravascular Epidural Catheter Insertion. Anesthesia \& Analgesia. August $2007: 105: 2: 460-464$

9. el-Behesy BA, James $\mathrm{D}$, Koh KF et al. Distinguishing cerebrospinal fluid from saline used to identify the extradural space. British Journal of Anaesthesia. 1996: Dec: 77(6): 784785.

10. Beilin Y, Arnold I, Telfeyan C, et al. Quality of analgesia when air versus saline is used for identification of the epidural space in the parturient. Regional Anesthesia Pain Medicine. 2000 Nov-Dec:25(6): 596-9.

11. Samuel Evron, Daniel Sessler, Oscar Sadan et al. Identification of the epidural space: Loss of resistance with air, Lidocaine, or the combination of air and Lidocaine. Anesthesia Analgesia 2004: 99: 245-50.

12. A. Patrick, C. Miller. More on epidural needle wings. Anaesthesia. 2006:61: 4: 405-406.

13. L.H.Hoffmann, M.P.Vercauteren, J.P. Vreugde et al. Posterior epidural space depth: safety of the loss of resistance and hanging drop technique. British Journal of Anaesthesia. 1999:83(5):807-9.

14. Baraka A. Identification of the peridural space by a running infusion drip. British Journal of Anaesthesia 1972: 44: 122.

15. Pan, Peter H. M.S.E.E, Sintay, Benjamin J. Eyes to the Needle: To Assist Identification of the Epidural Space. 2010: Volume 112: Issue 5: 1073-1075.

16. Oscar Ghelber, Ralf E. Gebhard, Sejal Vora et al. Identification of the Epidural Space Using Pressure Measurement With the Compuflo Injection Pump - A Pilot Study.
2008: volume 33: issue 4: pages 346-352. Regional Anesthesia and Pain Medicine.

17. Ting, Chien-Kun; Tsou, Mei-Yung; Chen, Pin-Tarng et al. A New Technique to Assist Epidural Needle Placement: Fiberoptic-guided Insertion Using Two Wavelengths. May 2010 : Volume 112: Issue 5: pages 1128-1135.

18. Jacob S, Tierney E. A dual technique for identification of the epidural space. Anaesthesia. 1997 Feb:52(2): pages 141-3.

19. Goodchild.G.s. and Noble J.The effects of intrathecal midazolam on sympathetic nervous system reflexes in man- a pilot study.Brit J Clin Pharmacol 1987; March 23(3)279.89.

20. Tony L. Yaksh and Jeffrey W. Allen, The Use of Intrathecal Midazolam in Humans: A Case Study of Process Anesth Analg 2004;98:153645

21. Malinowsky JM, Cozian A, Lepage JY, Mussini JM, inaud M, Souron R. Ketamine and midazolam neurotoxicity in the rabbit. Anesthesiology 1991; 75: 91-97.

22. Svensson BA, Welin M, Gordh T Jr, Westman J. Chronic subarachnoid midazolam (Dormicum) in the rat; morphologic evidence of spinal cord neurotoxicity. Reg Anesth 1995; 20: 426-434.

23. Tucker AP, Lai C, Nadeson R, Goodchild CS. Intrathecal midazolam I: a cohort study investigating safety. Anesth Analg. 2004; 98: 1512-20. 